\author{
APPENDIX I
}

\title{
REPORT OF THE COMMITTEE ON THE SPECTRA OF VARIABLE STARS
}

(prepared by M. W. Feast, Chairman)

The field covered by this report overlaps particularly with the work of Commissions 29 and 45 whose reports should be consulted. I have been greatly helped by reports on Soviet work by Professor Kukarkin and Dr Boyarchuk and a bibliography of European literature by Bloch.

\section{General}

The Sonneberg programme for determining spectral types of variables has been described by Götz and Wenzel $(A N, 290,99)$ and several lists have been published in $M V S$. Bakos is studying high dispersion spectra of a number of variables that are members of visual binary systems.

\section{Mira, semi-regular and RV Tauri variables}

Stephenson and Terrill $(A p J, 147,148)$ and Houk $(I B, 228)$ have given spectral types for a considerable number of long period variables. Terrill $(A J, \mathbf{7 4}, 413)$ has made an extensive study of the variation of spectrum with phase for 51 red variables. Deutsch, Keenan and Garrison have continued their extensive programme of spectral classification of Mira variables. Keenan and Dessy $(A p J, 146,583)$ suggest that the relatively early spectral type of the very long period Miras ( $\mathrm{R}$ Cen, $\mathrm{R}$ Nor etc.) showing double maxima indicates that the fundamental parameter is the half-period (between successive maxima). A similar conclusion $(A p J, 93,380 ; M N, 125,367)$ was reached earlier by others on the basis of the correlation with period of the difference between absorption and emission velocities. Feast has used $\mathrm{K}$ subgiant companion of $\mathrm{U}$ Men to derive an absolute magnitude of -0.5 for the Mira star. The radial velocity of the companion agrees with the absorption velocity of the variable near maximum (Obs.).

Herbig and Zappala have now discussed in greater detail the identification of certain emission lines in $\chi$ Cyg as the inverse predissociation spectrum of $\mathrm{AlH}(Z f A, 68,423)$. J. P. Swings reports that studies of the relative intensities of [FeII] and AlO emissions in Miras support the idea of stratification of the emitting layers. The Swings' have also discussed (Lunteren) the possibility of molecular fluorescence being of significance for these stars. Maehara reports the identification of two emission lines $(4178,4138)$ in $o$ Ceti as due to SiH. Fluorescence excitation in Miras has been discussed by Gauzit (Ann. Astr., 30, 55). Herbig (Liège, 1968, p. 353) has discussed the presence of forbidden emission lines in Miras and other cool stars.

The study of red variables, including Miras, in the infrared is being actively pursued in several places and has yielded very important results. This work has recently been reviewed by Spinrad and Wing (Ann. Rev. 7, 249).

Tsuji has made a detailed study of the Se variables R And and R Cyg. Strong circumstellar K I and $\mathrm{Rb} \mathrm{I}$ have been found. A rapid mass loss $\left(10^{-5} M_{\odot} / \mathrm{yr}\right)$ is deduced. This is further evidence that the $\mathrm{S}$ stars are short lived. $\mathrm{H} \alpha$ in Se variables is bifurcated (probably by overlying $\mathrm{ZrO}$ ) (Bretz, Trieste, 1966, p. 166). Although the "Potassium Flares" found in some otherwise normal stars are likely to be spurious $(P A S P, 79,351)$, Miss Bretz points out that the $\mathrm{K} \mathrm{I}$ emission lines she found in 1966 in $\chi$ Cyg occurred on several different nights and are quite narrow, indicating that they are stellar emissions. They did not appear at the (brighter) 1967 maximum. Catchpole and Feast are carrying out a spectroscopic survey of southern $\mathrm{C}$ and $\mathrm{S}$ stars (Henize and Wray Catalogues). A considerable number of variables are included in the programme. Stephenson ( $A p J$, 150, 543) has discussed the violet opacity in the C-S star V 372 Mon. In the C-S category, Catchpole (Pretoria) is studying UY Cen and Culver (Perkins) CY Cyg. Pik-Sin The (PASP, 80, 104) points out that objective prism spectra show $\mathrm{R}$ Vol and HD114586 (KZP, 1995) to be C and $\mathrm{S}$ type re- 
spectively. These classifications are confirmed by unpublished Radcliffe spectrograms. $R$ Vol showed $\mathrm{H} \alpha$ emission in May 1953. Boyarčuk is studying high dispersion spectra of semiregular variables.

VY CMa, an irregular M-type variable with several nebulous companions has been found to be an intense infrared object, and $\mathrm{OH}$ and $\mathrm{H}_{2} \mathrm{O}$ source and to be strongly polarized. Its spectrum is discussed by Herbig (Liège, 1969) and Hyland et al. $(A p J)$. It is remarkable for containing ScO, YO and TiO in emission. Herbig associates VY CMa with the HII region around NGC 2362 and proposes a detailed model with the star as a pre-main-sequence object though Hyland et al. prefer to regard it as highly evolved. The infrared radiation is believed to originate from dust near VY CMa. This is supported by the observation (Feast, Obs.), that in one of the nebulous companions (reflection nebulae) the red TiO bands are stronger than in VY CMa itself where they are filled in by an overlying continuum.

A detailed study of $\mu$ Cep (M 2Ia) is being made by Deutsch who has discovered a radial pulsation with a 1100 day period. Bond has confirmed with slit spectrograms the report by himself and Bidelman that the red variable V 422 Cen was like $R$ Aqr in showing strong nebular emission lines. The fact that the M 8.5 III star V $1398 \mathrm{Sgr}$ (in the direction of the galactic centre) has only a small light amplitude has been taken to imply a difference from late type stars near the sun (Spinrad, et al., $A J, 74,525$ ).

The spectrophotometric gradient of the RV Tau star AC Her has been studied by Alania (Abastumani, 36, 16) and a further spectroscopic study made of U Mon (Aliev, Sov. AJ, 10, 794).

\section{Cepheids}

Schneider has continued his work on the classification of Cepheids in the Barbier, Chalonge, Divan System (J.d'Obs., 50, 419; AA, 2, 195). This has led to new determinations of reddening for 20 Cepheids. Guseva and Tsarevsky $(P Z, 16,292)$ have attempted to put published spectral types of Cepheids onto a common system. Opolski uses their results to discuss the spectral typeperiod relation $(A c A, 18,515)$. Lloyd-Evans $(M N, 141,109)$ has made new radial velocity observations of cepheids and finds the frequency of occurrence of cepheid binary systems to be at least $15 \%$. V $636 \mathrm{Sco}, \mathrm{S}$ Mus and AW Per are spectroscopic binaries. The latter has a composite spectrum as does KN Cen. This work is being continued. From spectroscopic observations Carevsky and Vitričenko (Crimea) suspect the duplicity of the blue companion of $\delta$ Cep.

Rodgers and Bell have continued their high dispersion studies of cepheids $(M N, 135,121 ; 136,91$; 138, $23 ; 139,175 ; 142,161)$. They determine atmospheric parameters and fix the minimum width of the instability zone as $\Delta(B-V)=0.24$ at 10 day period. The effect of line blanketing has been studied and chromospheric effects have been found in $\mathrm{H} \alpha$ for $\beta$ Dor and (markedly) 1 Car. In the latter star one of the chromospheric $\mathrm{H} \alpha$ components is stationary suggesting a (non-pulsating) circumstellar shell. In the disk cepheid $\kappa$ Pav the $s$-process elements are deficient relative to $e$-process elements. A similar result is found for RT Aur by Bappu and Raghavan $(M N, 142,295)$. Caputo $(M A 1,39,401)$ has made a detailed analysis of $\zeta$ Gem whilst Breger $(M N, 136,61)$ determined radius variations for U Sgr. Wallerstein has begun a programme to study spectra of cepheids in the red region. With Grenfell he has determined line shift and depth effects in SV Vul and plans to work on T Mon. Kipper (Tartu, 36, 234) has made a curve of growth analysis of Polaris.

The carbon variable RU Cam continues to attract attention. Wallerstein and Crampton ( $A p J, 149$, $225)$ showed that when its light was nearly constant so was its radial velocity. Detailed spectroscopic analyses have been made by Faraggiana and Hack $(Z f A, 66,343)$ and Wallerstein $(A p J, 151,1011)$. $\mathrm{Fe} / \mathrm{H}$ is normal and $\mathrm{C} 12 / \mathrm{C} 13 \sim 9$. Blue shifted Ca II emission gives evidence of mass loss.

\section{RR Lyrae, Dwarf Cepheids, $\delta$ Scuti and $\beta$ CMa stars}

During the last few years detailed studies of $\delta$ Scuti stars from high dispersion spectra and spectral scans have considerably increased understanding of these stars (papers by Kuhi, Danziger, Dickens, Bessell etc., see ApJ Sup., 18, 167). Due to improvements in the absolute spectrophotometric 
calibration and in blanketing corrections Bessell's conclusions differ significantly from earlier work. In particular he determines much higher masses $\left(\sim 2 M_{\odot}\right)$ and concludes that these are old A stars evolving off the main sequence. Unlike most pulsating stars some $\delta$ Scuti variables have high rotational velocities (cf. Astr. Lett., 1, 213). Bessell finds from a curve of growth analysis that $\delta$ Sct, $\delta$ Del and $\varrho$ Pup are similar to the Am stars in microturbulence and abundances. However Reimers $(A A, 3,94)$ finds different relative abundances for $\delta$ Del using a model atmosphere approach and connects this star with the Ap and $\lambda$ Boo stars. The Am star 28 And appears to show $\delta$ Sct type variations (Nishimura, Astr. Space Sci., 3, 77). Further radial velocity observations have been obtained of 4 CVn (PASP, 79, 330) and 14 Aur (Astr. Lett., 2, 175).

Bessell has also studied the AI Vel stars (Dwarf Cepheids). Slight metal under-abundances and low masses $\left(\sim 0.5 M_{\odot}\right)$ are found and he considers them as posthelium-flash stars. The high lithium abundance of $\mathrm{VZ}$ Cnc which presumably belongs to this class is notable $(A p J, 147,151)$ (see also C.R., 267B, 657).

Preston and Spinrad $(A p J, 147,1025)$ investigated the 90 day cycle of the RR Lyrae star RV UMa. No stillstand phenomenon was found. In BL Her (1.3 day period) $\mathrm{H}$ emission and $(U-B)$ excess correlate as for RR Lyraes but the phase lag of luminosity relative to velocity is different (Preston and Kilston, $A p J, 148,787)$. Values of the $\Delta s$ parameter for RR Lyraes were determined by Alania (Abastumani, 36, 24) and Clube et al. (Mem. RAS, 32, 101) who also give radial velocities.

Orlov (Soviet $A J, 12,89,450$ ) finds no apparent variation with time of the equivalent widths or central intensities of $\mathrm{H}$ and He lines in the $\beta \mathrm{CMa}$ stars, $\gamma$ Peg and $\delta$ Cet. Periodic changes of equivalent width of some lines in $\delta$ Cet are however reported by Ciurla and in $\gamma$ Peg by Contel (Budapest, p. 297). The rotational velocity of RS Sex is high $(I B, 260)$. Breger $(M N, 136,51)$ discussed $\alpha$ Lup and $\beta$ Cen and van Hoof (Toronto, p. 237) $\theta$ Oph.

\section{5. $R$ CrB, symbiotic and Nova-like variables}

Observers at the Cape and Radcliffe Observatories have obtained extensive photometric and spectroscopic observations of the R CrB star RY Sgr during and since the 1967 minimum. Early results (Budapest, p. 253) show a very rich emission spectrum during decline, a blue continuum (interpreted as the electron attachment spectrum of $\mathrm{CN}$ ), evidence for rapid mass ejection and for heavy reddening during minimum. The star has a marked infrared excess (Lee, Feast, $A p J, 157$, L173) as does R CrB itself (Stein et al., ApJ, 155, L3). A periodicity of about 39 days found in the light and colours is being sought in the radial velocities. Greenstein noted weak chromospheric emission in $\mathrm{R} \mathrm{CrB}$ during a shallow minimum (IAU Cir., 2158). At the University of Western Ontario, Gray, Moorhead and Marlborough are working on spectroscopic problems of $\mathrm{R} \mathrm{CrB}$ stars. Abundances in R CrB, XX Cam and RY Sgr have been determined. A detailed spectroscopic description of Herbig's Cin emission object V 348 Sgr (which may be related to the $\mathrm{R} \mathrm{CrB} \mathrm{stars)} \mathrm{has} \mathrm{been} \mathrm{given}$ by Houziaux $(B A C, 19,265)$.

Problems of the symbiotic stars have been reviewed by Boyarčuk (Budapest, p. 395) and Sahade (Liège, 1968, p. 309). There is considerable interest in these stars at several observatories in the USSR (Soviet AJ, 10, 331; 783; 11, 8; 453; Tartu, 35, 188, Izv. Krym, 38, 155. Astrofizika, 2, 50, 103). Line intensities and continuum energy distributions have been determined in $Z$ And, AG Peg and AG Dra. Boyarčuk finds that on plates taken by Herbig in 1961 during a flare $Z$ And resembles an A3 P Cygni type. MH $\alpha 208-51$ is a symbiotic object $(I B, 254)$. TX CVn has been briefly discussed (Padova Comm., 46). The general mode of excitation in symbiotic stars is discussed by llovaisky and Wallerstein $(P A S P, 80,155)$. Bloch et al. have a study of $\mathrm{Z}$ And in press and Aller and Wares have observed RY Mus. Friedjung has investigated AG Peg in detail $(A A)$ whilst HD 4174 and WY Gem have been described (MAI, 39, 633; 40, 25).

MH $\alpha$ 328-116 (= V1016 Cyg) continues to be observed. The lines of higher ionization are strengthening (Liège, 1968, p. 363, 369; Ap. Lett., 3, 141, IB, 372). The underlying continuum in 1965-66 corresponded to a very hot star (O'Dell, $A p J, 149,373$ ). An object of possibly similar type is HBV 475 recently discovered by Kohoutek (IB, 384, IAU Circ., 2174, 2176, 2182). 
In June 1967 Deutsch found that CH Cyg had undergone another outburst and that a hot continuum was present. The spectrum has been extensively studied by Faraggiana and Hack (Liège, 1968, p. 317; Trieste, 1968, p. 336). There is evidence for mass loss. The system seems to consist of an $M$ giant and an unstable blue subdwarf. Similar conclusions were reached by Luud (Tartu). There is also evidence for long period orbital motion (Deutsch, Hack) and rapid fluctuations in the ultraviolet continuum (e.g. Walker et al., $A p J, 156,117$ ). Swings discussed the ultraviolet line spectrum (Astr. Lett., 1, 54).

$\eta$ Car continues to receive much attention. The infrared continuum (apparently thermal) is exceptionally strong (at 10 and $20 \mu$ it is the brightest object outside the solar system, Westphal and Neugebauer $A p J, 156$, L45). Pagel and Lambert (Nature, 221, 325; 223, 726) used the [FeII] lines to determine a much higher reddening than previously supposed. They consider that the visual continuum may well be due to two photon emission from hydrogen. Viotti (Astr. Space Sci., 5, 323) has investigated the absolute magnitude, the continuum and spectral variations whilst Thackeray has discussed the line in the $9000 \AA-11300 \AA$ region (Liège, 1968, p. 323; MNASSA, 28, 37; MAI, 38, $451 ; 39,105$ ). Aller has also worked on $\eta$ Car. Thackeray (Liège, 1968, p. 327) has described the progressive ionization (up to [FevII] in RR Tel and other forbidden lines. Evidence for [NiIv] has been strengthened (Lambert and Thackeray, Astr. Space Sci., 5, 283) and [FeIv] has been rediscussed on the basis of laboratory work (Edlen, $M N, 144,391$ ).

Herbig and Boyarčuk $(A p J, 153,397)$ find the spectrum of the strange variable FG Sge changed from B4 I to A5 Ia between 1955 and 1965. This is interpreted as the ejection of a nebular shell from an (invisible) hot subluminous star. The nebula around the star is probably the remnant of a previous ejection (see also $P A S P, \mathbf{8 0}, 712 ; \mathbf{8 1}, 274$ ). AG Carinae is being studied by Viotti and Caputo.

\section{Flare stars, $T$ Tauri stars and related objects}

Objective prism studies of some T Tauri stars have been carried out by Götz and Wenzel (MVS, 4, 5). Hydrogen emission in $T$ Tauri stars has been discussed by Anderson, Kuhi, Dibaj and Esipov whilst Walker has shown that matter falls into the UV excess stars at a variable rate (Budapest, p. 93, 103, 107). Infrared Ca II emission in MWC 1080 suggests that this may be a T Tauri star (MacConnell, $P A S P, 79,66)$.

Haro and others discuss the spectra of flare stars (Ton. Bul., 31) and suggest that the intensity of the Ca II emission lines in $\mathrm{G} \mathrm{8-K} 0$ or later types is a good criterion for predicting flare activity. Kunkel $(A p J)$ has obtained time resolved spectra of dMe flares. He finds that these flares cover only a few percent of the stellar surface despite the large energy output. Kandel (Trieste, 1966, p. 146) discusses emission in HD 119850. Wolf 359 (dM8e) was observed spectroscopically during a flare (Greenstein and Arp, Astr. Lett., 3, 149) Gershberg (Izv. Krym., 38, 177) has analysed simultaneous spectroscopic and photometric observations of flare stars. Thackeray has classified Butler's flare star as dKO (see Andrews $P A S P, 79,368$ ).

Herbig $(A p J, 152,439)$ has drawn attention to the fact that $\mathrm{R}$ Mon is a non-stellar object and that there is no convincing spectroscopic evidence for the presence of a $\mathrm{G}$ or $\mathrm{K}$ type star, the absorption spectrum sometimes seen being that of a transient shell.

\section{Novae}

A large number of observational papers have been published on recent novae especially of the peculiar slow Nova Del 1967. Much more material is unpublished. It remains a problem that so little of the available material is ever taken past the purely descriptive stage (if even as far as that). A group has formed at the Institut d'Astrophysique (Paris) specifically to study nova spectra.

Nova Del 1967. A pre-outburst, objective prism observation shows an early type continuum (Stephenson, $P A S P, 79,584)$. It is not possible to list all the papers and work on this object. There have been extensive observations at Lick (cf. Sky Tel., 35, 77) Victoria (cf. Trieste, 1968, p. 290), Haute Provence (cf. AA, 1, 403; Trieste, 1968, p. 281), Asiago and elsewhere. 
Nova Vul 1968, has been described by Mammano et al. (Budapest, 1968, p. 271), Bloch (Haute Provence, 10, no. 13) and Dorschner et al. (AN, 291, 217).

Nova Vul 1968 no. 2, has been described by Rosino et al. (Astr. Space Sci., 4, 392).

Nova Her 1963, Doroschenko has made a detailed study of this nova (Soviet AJ, 12, 95; Astrofizika, 2, 240). Tremko (Skalnate) is working up Hamburg spectra and Friedjung (Paris) is studying the Balmer absorption line profiles (see also Tartu Pub., 36).

Nova Her 1960. Work on this nova has been published by Hidajat $(Z f A, 68,164)$, Luud and Kuningas (Tartu Pub., 35, 413) and Savel'eva (Soviet $A J, 11,576$ ).

General. Antipova (Astr. Zu., 46, 366) considers the great strength of $\mathrm{CN}$ in DQ Her at outburst as an abundance effect in the shell.

Recurrent Novae. The equivalent widths of the coronal lines in $\mathrm{T} \mathrm{CrB}$ have been measured by Bochonko and Malville $(P A S P, 80,177)$ and the radius and density of the coronal envelope determined. The 1967 outburst of T Pyx was described by Catchpole (MN, 142, 119 and Rosino et al. (Budapest, p. 261). Tolbert, Pecker and Pottasch have reduced a considerable number of plates of RS Oph at its 1958 outburst and Pottasch has proposed a detailed quantitative model $(B A N, 19,17$, 227). Spectra of the star between the 1958 and 1967 outbursts have been described by Wallerstein and Cassinelli (PASP, 80, 589) and Barbon et al. (Budapest, p. 257) whilst Code (ApJ, 151, L149) described 1967 spectra.

\section{Pulsars, $X$-ray sources, hot subluminous variables}

NP0532 (the Crab Nebula pulsar) has been investigated by a number of workers (e.g. Lynds, $A p J, 157$, L11) who, like Minkowski earlier, find the spectrum to be continuous. Oke ( $A p J, 156$, L49), from spectral scans finds the energy distribution to be the same in the main and the interpulse and to be well fitted in the visual region by a Planck function at $10000 \mathrm{~K}$. The measurements have been extended into the infrared by Neugebauer et al. Westphal et al. (ApJ, 155, L109) show that the star near CP 1919 is almost certainly not associated with the pulsar.

The most detailed spectroscopic study of Sco X-1 so far is probably that of Westphal et al. ( $\mathrm{ApJ}$, 154, 139). There is a hot continuum with $\mathrm{H}, \mathrm{HeI}$ and II and other emission lines. The spectrum has a general resemblence to that of old novae and $U$ Gem stars. Line strengths relative to the continuum and radial velocities vary in a complex manner which is not yet properly understood. The object may be a spectroscopic binary but this has not been proved. Spectrophotometry has been published ( $A p J, 149, \mathrm{~L} 45 ; 155,1)$. The spectrum of Cyg X-2 is quite complex (Lynds, Burbidge, Sandage, Kraft etc. $A p J, 149$, L41 ; 150, L95; L99; L83; 155, L159). HeI and II emissions are present and an absorption component which Kraft and Demoulin identify as probably a $\mathrm{G}$ subdwarf. Some of the absorption lines appear to be formed in gas streams. Large velocity variations have been found but it has not been conclusively demonstrated that the star is a binary though spectrophotometric scans suggest the presence of a hot continuum ( $A p J, 151, \mathrm{L93})$. Cen XR-2 seems almost certainly identified with the rapid irregular variable WX Cen whose spectrum resembles Sco X-1 (Eggen et al., $A p J, 154$, L27). HD 310376 (Schild, $A p J, 157,709$ ) appears to have many similarities to Sco X-1 but is not known as an $\mathrm{X}$-ray source. The spectrum varies from night to night. In addition to emission lines, $\mathrm{H}$ and $\mathrm{He}$ absorptions are sometimes seen.

The central star of the nebulosity NGC5189 is a WR star with strong Ovi 3811, 3834 ( $A p J, 152$, L135 and Radcliffe, unpublished). Because the star was an early (but now unlikely) identification for Cen XR-2 it is interesting that a spectroscopically similar object has been found close to the position of $\mathrm{GX} 3+1$ (Freeman et al., Nature, 219, 251; Blanco et al., ApJ, 152, L137). Sanduleak (PASP, 80, 470; IAU Circ., 2150) has found another two similar high excitation WR stars (one in the SMC).

Walker and Chincarini $(A p J, 154,157)$ find that at minimum in SS Cyg the irregular light variations result entirely from changes in the intensity of the continuum, the emission line intensities being unchanged. The outbursts are shown to be associated with the hot star around which there is probably a rotating ring of matter. Spectrophotometry of SS Cyg at the time of a flare is described 
by Chalonge et al. (Haute Provence, 10, no. 9). This is the first of a series of such investigations on unstable stars. Bartaya (Astrofizika, 2, 249) also obtained spectrophotometric observations of SS Cyg during flares. Kraft et al. ( $A J, 73, \mathrm{~S} 21)$ find $\mathrm{Z}$ Cam to be a short period double lined binary (sdBe $+\mathrm{G}($ ?)) with both stars of about solar mass. Lortet-Zuckermann (Budapest, p. 381) finds broad $\mathrm{H}$ absorption during a stillstand of this star which she attributes to Stark broadening in the atmosphere of a dwarf $\mathbf{G}$ star.

BL Lac has been studied intensively since Shmitt (Nature, 218, 663) pointed out that it coincided with the peculiar radio source VRO 42.22.01 ( $A p J, 155, \mathrm{~L} 133 ; 156, \mathrm{~L} 41, \mathrm{~L} 135 ;$ IAU Cir., 2170). The spectrum appears to be continuous and may be a continuation of the radio synchrotron radiation. The continuum is highly polarized. As the star brightens the continuum reddens. A possible (unidentified) emission line has been found by Oke et al. It is not yet clear whether the object is galactic or extragalactic.

\title{
APPENDIX II
}

REPORT OF THE COMMITTEE ON VARIABLE STARS IN CLUSTERS

(prepared by Mrs. H. B. Sawyer-Hogg, Chairman)

\author{
Committee Members: M. W. Feast, G. H. Herbig, L. Rosino
}

Because of space restrictions, this report on the variable stars in clusters differs in form from those in earlier IAU reports, and is more condensed. Not all of the papers which have appeared since the last IAU General Assembly can be mentioned.

A definite trend is apparent in this field. A few years ago the aim of many programs was to detect new variables or to determine the periods of those known. While this is still being carried on, especially for the clusters in the far south, the emphasis has subtly changed to an avid pursuit of information about individual variables. For many variables, much more is known now than just the periodicity of their magnitude variations in photographic light. The number of observers studying these stars and the number of telescopes used in the program have greatly increased.

\section{Variables in globular clusters}

\section{(a) Catalogues}

The number of clusters searched for variables has now crossed the hundred mark. Of approximately 130 clusters considered to be globular, in and around our galaxy, 102 have now been examined to some degree. In these, more than 1900 variables have been found, but 10 clusters have no variables. The Atlas y Catalogo de Estrellas Variables en Cumulos Globulares al sur $\mathrm{De}-29^{\circ}$ by Fourcade, Laborde and Albarracin (Cordoba, 1966) appeared about the time of the last IAU and is of inestimable value for these little known southern clusters. The third catalogue of variables for all globular clusters by Sawyer-Hogg with references should appear in the David Dunlap Publications at about the same time as this report. A summary was given at the IAU IVth Colloquium on Variable Stars, Budapest, 1968 and appeared in this publication (Budapest, p. 475).

\section{(b) Discovery of new variables}

A cluster near the south celestial pole, IC4499, has come into prominence with the announcement by Fourcade and Laborde (MAI) that they have found 128 variables in it, with 42 other stars suspected of variation. This places IC4499 on the list of clusters richest in variables, exceeded only by M 3 and Omega Centauri. Most of the variables are of short period, and are probably RR Lyrae stars. IC 4499 is a loose globular cluster, class XI, ang. diam. 6.2.

In addition to this cluster, Fourcade and Laborde have found a few variables in each one of a 